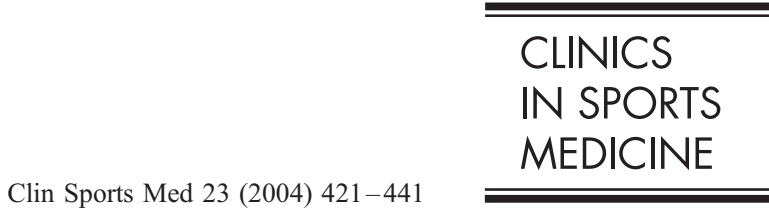

\title{
Return to play following sports-related concussion
}

\author{
Mark Lovell, $\mathrm{PhD}^{\mathrm{a}, \mathrm{b}, \mathrm{c}}$, Micky Collins, $\mathrm{PhD}^{\mathrm{a}}$, \\ James Bradley, $\mathrm{MD}^{\mathrm{a}, \mathrm{d}, *}$ \\ ${ }^{\mathrm{a}}$ Sports Medicine Concussion Program, Department of Orthopaedic Sugery, \\ University of Pittsburgh Medical Center, 200 Delafield Road, Suite 410, Pittsburgh, PA 15215, USA \\ ${ }^{\mathrm{b}}$ National Football League Neuropsychological Testing Program, Pittsburgh, PA 15215, USA \\ ${ }^{\mathrm{c}}$ National Hockey League Neuropsychological Testing Program, Pittsburgh, PA 15215, USA \\ ${ }^{\mathrm{d}}$ Team Physician, Pittsburgh Steelers, Pittsburgh, PA 15215, USA
}

The diagnosis and management of sports-related concussion can be one of the most challenging endeavors for the sports medicine practitioner. The team physician is often called upon to make the return to play decision based on limited observation of the athlete and a brief sideline evaluation. Furthermore, return to play decisions are often made against the backdrop of a noisy stadium or arena where there is intense pressure from coaches, fans, and other players to return the injured athlete to the playing field as quickly as possible.

As a result of concerns regarding both short-term and potential long-term sequelae of concussion, more than 20 management guidelines have been published to provide guidance regarding return to play issues. However, in the absence of supporting research to substantiate any particular return to play protocol, concussion management strategies throughout the 1980s and 1990s were based on the opinion of individual physicians or groups of experts rather than on empirical findings $[1,2]$. However, current research has prompted a reevaluation and revision of prior guidelines along with recent revisions in concussion management strategies. This article reviews new developments in the evaluation and management of sports-related concussion and focuses specifically on practical suggestions for making return to play decisions.

\section{Definition of concussion}

Despite hundreds of studies and years of research, there is currently no universally accepted definition of concussion. However, for the past three decades

\footnotetext{
* Corresponding author.

E-mail address: bradleyjp@msx.upmc.edu (J. Bradley).
} 
the definition originally proposed by the Committee on Head Injury Nomenclature of Neurological Surgeons in 1966 has by far represented the most popular definition. This committee defined concussion as "a clinical syndrome characterized by the immediate and transient post-traumatic impairment of neural function such as alteration of consciousness, disturbance of vision or equilibrium, etc., due to brain stem dysfunction" [3].

More recently, however, other definitions of concussion have been posed. For example, the American Academy of Neurology (AAN) defines concussion as "any trauma induced alteration in mental status that may or may not include a loss of consciousness" [4].

Authors of the AAN definition felt the Committee on Head Injury Nomenclature definition may be too limiting, because other brain structures (eg, cortical areas) are commonly associated with concussion and the injury is not limited to the brain stem. Along this same vein, authors of the AAN guidelines wished to highlight the fact that concussion may occur with or without a loss of consciousness.

\section{Pathophysiology of concussion}

Recent research into the subtle metabolic effects of concussion has triggered burgeoning insights into the pathophysiology of concussion. Using a rodent model, Hovda and colleagues [5] have described a metabolic dysfunction that occurs when cells immediately injured upon concussive insult are exposed to dramatic changes in both their intracellular and extracellular environments. These changes are the result of excitatory amino acid-induced ionic shifts with increased $\mathrm{Na} / \mathrm{KATP}$-ase activation and resultant hyperglycolysis [6]. Thus there is a high-energy demand within the brain shortly after concussive injury. This process is accompanied by a decrease in cerebral blood flow that is not well understood, though it may be secondary to accumulation of endothelial $\mathrm{Ca}^{++}$, which is thought to cause widespread cerebral neurovascular constriction. The resulting "metabolic mismatch" between energy demand and energy supply within the brain has been postulated to propagate a cellular vulnerability that is particularly susceptible to even minor changes in cerebral blood flow, increases in intracranial pressure, and apnea. Animal models have indicated that this dysfunction can last up to 2 weeks or theoretically longer in the human model [5].

Given that concussion is a metabolic rather than structural injury, traditional neurodiagnostic techniques (eg, CT, MRI, neurologic exam) are almost invariably normal following concussive insult [7]. It should be stressed, however, that these techniques are invaluable in ruling out more serious pathology (eg, cerebral bleed, skull fracture) that may also occur with head trauma.

Understanding the metabolic process of concussion in the human model is premature, but this model of pathology raises many questions regarding the threat of vulnerability, how long it lasts, and the potential synergistic effects of multiple injuries. It has been postulated that metabolic dysfunction, until fully resolved, 
may lead to significantly increased neurologic vulnerability if a subsequent trauma, however minor, is sustained. Such metabolic dysfunction is theoretically linked to second impact syndrome [8] and may also form the basis for the less severe, though occasionally incapacitating, presentation of postconcussion syndrome. Although long-term deficits in the form of postconcussion syndrome have been observed from a single concussive event, it is believed that proper management of concussive injury should lead to good prognosis and minimal deleterious effects with regard to brain function. Conversely, returning an athlete to participation before complete recovery may greatly increase the risk of lingering, long-term, or even catastrophic neurologic sequelae. As such, acute assessment of concussion and determination of any existing signs or symptoms of injury proves critical to the safe management of the athlete.

\section{On-field and sideline management of concussion}

The diagnosis of cerebral concussion can be tricky under the best of circumstances. There may be no direct trauma to the head, and the concussed athlete is infrequently rendered unconscious. The athlete may be unaware that they have been injured immediately after the injury and may not show any obvious signs of concussion such as clumsiness, gross confusion, or obvious amnesia. To complicate the situation, athletes at all levels of competition may minimize or hide symptoms in an attempt to prevent their removal from the game, thus creating the potential for exacerbation of their injury.

\section{Initial sideline signs and symptoms of evaluation and return to play}

Box 1 provides a summary of common on-field signs and symptoms of concussion. It should be stressed that sideline presentation may vary widely form athlete to athlete, depending on the biomechanical forces involved, specifically affected brain areas, prior history of injury, and numerous other factors. In reviewing the common signs and symptoms of concussion, it is imperative to note that an athlete may present with only one symptom or potentially a myriad of symptoms. A thorough assessment of all common symptoms associated with concussion should be conducted with the concussed athlete.

Headache is the most commonly reported symptom of injury and may be seen in up to $70 \%$ of athletes who sustain a concussion [9]. Though it is true that musculoskeletal headaches and other pre-existing headache syndromes may complicate the assessment of postconcussion headache, any presentation of headache following a blow to the head or body should be managed conservatively. Most frequently, a concussion headache is described as a sensation of pressure in the skull that may be localized to one region of the head or may be generalized in nature. In some athletes, particularly those with a history of migraine, the headache may take the form of a vascular headache, may be unilateral, and is often 


\section{Box 1. University of Pittsburgh signs and symptoms of concussion}

Signs observed by staff

Appears to be dazed or stunned

Is confused about assignment

Forgets plays

Is unsure of game, score, or opponent

Moves clumsily

Answers questions slowly

Loses consciousness

Shows behavior or personality change

Forgets events prior to play (retrograde)

Forgets events after hit (posttraumatic)

\section{Symptoms reported by athlete}

Headache

Nausea

Balance problems or dizziness

Double or fuzzy/blurry vision

Sensitivity to light or noise

Feeling sluggish or slowed down

Feeling "foggy" or groggy

Concentration or memory problems

Change in sleep pattern (appears later)

Feeling fatigued

described as throbbing or pulsating. The headache may not develop immediately after injury and may develop in the minutes or even hours following injury. Therefore, it is essential to question the potentially concussed athlete regarding the development of symptoms beyond the first few minutes after injury. Most commonly, postconcussion headache is worsened with physical exertion. Thus if the athlete complains of worsening headache during exertional testing or return to play, postconcussion headache should be suspected, and conservative management is indicated. Although headache following a concussion does not necessarily constitute a medical emergency, a severe or progressively severe headache, particularly when accompanied by vomiting or rapidly declining mental status, may signal a life-threatening situation such as a subdural hematoma or intracranial bleed. This should prompt immediate transport to hospital and a CT scan of the brain.

Although headache is the most common symptom of concussion, concussion may occur without headache, and other signs or symptoms of injury should be carefully detailed and assessed. For example, athletes will commonly experience 
blurred vision, changes in peripheral vision, or other visual disturbances. These visual changes, in addition to photosensitivity or balance problems, are commonly associated with a blow to the back of the head (eg, head to turf). Moreover, an athlete may report increased fatigue, "feeling a step slow," or feeling sluggish. Fatigue is especially prominent in concussed athletes in the days following injury, and this symptom may be as prominent as headache in this regard. In addition to these symptoms, cognitive or mental status changes are commonly seen immediately following injury. Athletes with any degree of mental status change should be managed conservatively, and a thorough discussion of these issues is warranted.

\section{On-field evaluation of mental status and markers of concussion}

Appropriate acute care of the concussed athlete begins with an accurate assessment of the gravity of the situation. As with any serious injury, the first priority is always to evaluate the athlete's level of consciousness and ABCs (airway, breathing, and circulation). The attending medical staff must always be prepared with an emergency action plan in the event that the evacuation of a critically head- or neck-injured athlete is necessary. This plan should be familiar to all staff, well delineated, and frequently rehearsed.

Upon ruling out more severe injury, acute evaluation continues with assessment of the concussion. First, the clinician should establish the presence of any loss of consciousness (LOC). By definition, LOC represents a state of brief coma in which the eyes are typically closed and the athlete is unresponsive to external stimuli. LOC is relatively rare and occurs in less than $10 \%$ of concussive injuries. Moreover, prolonged LOC $(<1-2$ minutes) in sports-related concussion is much less frequent [10], and athletes with LOC are typically unresponsive for only a brief period. Obviously, any athlete with documented LOC should be managed conservatively, and return to play is contraindicated.

A more common form of mental status change following concussion involves confusion and amnesia. Confusion (ie, disorientation), by definition, represents impaired awareness and orientation to surroundings, though memory systems are not directly affected. Confusion is often manifested by the athlete appearing stunned, dazed, or glassy-eyed on the sideline. Confusion is frequently revealed in athletes who have difficulty with appropriate play-calling, answer questions slowly or inappropriately, or repeat oneself during evaluation. Teammates are often the first to recognize that an athlete has been injured given the level of disorientation and difficulty in maintaining the flow of the game. Upon direct evaluation by the physician or athletic trainer, the athlete may be slow to respond. To properly assess the presence of confusion, simple orientation questions can be directed to the athlete (eg, name, current stadium, city, opposing team, current month and day).

A careful evaluation of amnesia is of paramount importance in the concussed athlete. Amnesia may be associated with loss of memory for events preceding or 
following injury. Specifically, posttraumatic or anterograde amnesia is typically represented by the length of time between trauma (eg, helmet-to-helmet contact) and the point at which the individual regains normal continuous memory functioning (eg, standing on the sideline after the hit). As outlined in Box 1, on-field posttraumatic amnesia may be assessed through immediate and delayed (eg, 0, 5, 15 minutes) memory for three words (eg, girl, dog, green). It should be noted that confusion and anterograde amnesia are not mutually exclusive and may be difficult to dissociate. To help clarify this issue, anterograde amnesia represents a loss in memory from the point of injury until the return of a full, ongoing memory process. Confusion in and of itself is not associated with memory loss. These two markers of injury may be properly assessed once the athlete's confusion is cleared and lucid mental status returns. At that point, simply asking the athlete to recall the specific events that occurred immediately subsequent to trauma (eg, memory of returning to sideline, memory of subsequent plays, memory of later parts of the contest). Any failure to properly recall these events is indicative of anterograde amnesia. Any presence of amnesia, even in seconds, has been found to be highly predictive of postinjury neurocognitive and symptom deficit [10].

Retrograde amnesia, though given less focus in the literature, is also an important injury severity marker of concussion. Retrograde amnesia is defined as the inability to recall events occurring during the period immediately preceding trauma. To properly assess on-field retrograde amnesia, the athlete may be asked questions pertaining to details occurring just before the trauma that caused the concussion. As Box 2 highlights, asking the athlete to recall details of the injury is a good staring point. From there, asking the athlete to recall the score of the game before the hit, events occurring in the plays preceding the injury, and events occurring in the first quarter or earlier in practice is a practical assessment strategy. It should be noted that the length of retrograde amnesia will typically "shrink" over time. For example, as recovery occurs, the length of retrograde amnesia may contract from hours to several minutes or even seconds - though, by definition, a permanent loss of memory preceding injury occurs. Once again, even seconds of retrograde amnesia may be considered pathognomonic and predictive of outcome.

\section{Recent research on predictors of outcome following concussion}

Two published studies exist examining on-field predictors of outcome following sports-related concussion. Collins and colleagues [10] investigated the relationship between on-field markers of concussion severity and postinjury neurocognitive performance and symptom presentation in a group of 78 concussed high school and college athletes. Data from this study revealed that the presence of amnesia, not brief loss of consciousness, was most predictive of postinjury difficulties at 3 days postinjury. For example, athletes demonstrating "poor" presentation (notable cognitive deficits and high degree of symptoms) were over 10 times more likely to have exhibited any degree of retrograde 
Box 2. University of Pittsburgh sideline mental status testing card: on-field cognitive testing

\section{Orientation}

Ask the athlete the following questions:

- What stadium is this?

- What city is this?

- Who is the opposing team?

- What month is it?

- What day is it?

\section{Posttraumatic amnesia}

Ask the athlete to repeat the following words: girl, dog, green

\section{Retrograde amnesia}

Ask the athlete the following questions:

- What happened in the prior quarter or half?

- What do you remember just prior to the hit?

-What was the score of the game prior to the hit?

- Do you remember the hit?

\section{Concentration}

Ask the athlete to do the following:

- Repeat the days of the week backward, starting with today

- Repeat these numbers backward: 63, 419

\section{Word list memory}

Ask the athlete to repeat the three words mentioned earlier (girl, dog, green)

amnesia following concussion when compared with athletes with good postinjury presentation (no cognitive deficits and no reported symptoms at 3 days postinjury). Similarly, athletes with "poor" presentation were over four times more likely to have exhibited any degree of anterograde amnesia. Interestingly, loss of consciousness was not predictive of deficits following sports-related concussion. Similarly, a second outcome study [11] examined 47 athletes with concussion. These researchers found that athletes reporting memory problems at follow-up 
examinations had significantly more symptoms in general, longer duration of symptoms, and significant decreases on neurocognitive test performance. Data from these two studies directly contradict existing grading systems of concussion, which all delineate severity of injury and length of time until return to play on the LOC construct. The outlined data suggest that amnesia, and in particular retrograde amnesia, may be much more predictive in this regard.

\section{Return to play once concussion has been diagnosed}

\section{Return to play guidelines}

During the past 30 years, over 20 concussion management guidelines have been published with the intent of providing guidance and direction for the sports medicine practitioner in making complex return to play decisions [1]. These guidelines also provide accompanying grading scales designed to reflect and characterize the severity of the injury. Although these guidelines have no doubt resulted in improved care of the athlete, these multiple directives also create significant confusion and spark continuous debate. A historical review of all past and current concussion guidelines is beyond the scope of this article; however, a brief review of four of the more recent and popular guidelines is provided in Table 1.

Cantu [12] originally proposed his grading scale and management guidelines based on clinical experience, but was careful to emphasize that these guidelines were intended to supplement rather than replace clinical judgment. The original Cantu guidelines allowed return to play the day of injury if the athlete was symptom-free both at rest and following physical exertion. For athletes who experienced any loss of consciousness (eg, grade 3 concussion), a restriction of contact for 1 month was recommended. Athletes who had suffered a grade 2 concussion were allowed to return to play in 2 weeks if asymptomatic for a period of 7 days.

The Colorado Guidelines [13] were published in 1991 following the death of a high school athlete due to second impact syndrome and were drafted under the auspices of the Colorado Medical Society. These guidelines allowed for same-day return to play if symptoms cleared within 20 minutes of injury. For more severe injury (grade 3 concussion), the guidelines recommended immediate transport to a hospital for further evaluation. The guidelines were later revised under the sponsorship of the AAN [4]. The AAN guidelines allowed return to competition the same day of injury if the athlete's signs and symptoms cleared within 15 minutes of injury. Grade 2 concussion was managed in a manner similar to the Colorado guidelines, with return to competition within 1 week if asymptomatic.

More recently, Cantu has amended his guidelines [14] to emphasize the duration of posttraumatic symptoms in grading the severity of the concussion and making return to play decisions. Grade 1 concussion was redefined by an absence of loss of consciousness and postconcussion signs or symptoms lasting less than 
Table 1

Recent concussion grading scales

\begin{tabular}{|c|c|c|c|}
\hline Guideline & Grade 1 & Grade 2 & Grade 3 \\
\hline Cantu [6] & $\begin{array}{l}\text { 1. No loss of } \\
\text { consciousness } \\
\text { 2. Posttraumatic } \\
\text { amnesia lasts less } \\
\text { than } 30 \text { minutes }\end{array}$ & $\begin{array}{l}\text { 1. Loss of } \\
\text { consciousness } \\
\text { lasts longer than } \\
5 \text { minutes } \\
\text { OR } \\
\text { 2. Posttraumatic } \\
\text { amnesia lasts longer } \\
\text { than } 30 \text { minutes }\end{array}$ & $\begin{array}{l}\text { 1. Loss of consciousness } \\
\text { lasts longer than } \\
5 \text { minutes } \\
\text { OR } \\
\text { 2. Posttraumatic amnesia } \\
\text { lasts longer than } \\
24 \text { hours }\end{array}$ \\
\hline Colorado [23] & $\begin{array}{l}\text { 1. Confusion } \\
\text { without amnesia } \\
\text { 2. No loss of } \\
\text { consciousness }\end{array}$ & $\begin{array}{l}\text { 1. Confusion with } \\
\text { amnesia } \\
\text { 2. No loss of } \\
\text { consciousness }\end{array}$ & $\begin{array}{l}\text { 1. Loss of consciousness } \\
\text { (of any duration) }\end{array}$ \\
\hline $\begin{array}{r}\text { American Academy } \\
\text { of Neurology [1] }\end{array}$ & $\begin{array}{l}\text { 1. Transient } \\
\text { confusion } \\
\text { 2. No loss of } \\
\text { consciousness } \\
\text { 3. Concussion } \\
\text { symptoms, mental } \\
\text { status changes } \\
\text { resolve in less } \\
\text { than } 5 \text { minutes }\end{array}$ & $\begin{array}{l}\text { 1. Transient confusion } \\
\text { 2. No loss of } \\
\text { consciousness } \\
\text { 3. Concussion } \\
\text { symptoms, mental } \\
\text { status change lasts } \\
\text { longer than } 15 \text { minutes }\end{array}$ & $\begin{array}{l}\text { 1. Loss of consciousness } \\
\text { (brief or prolonged) }\end{array}$ \\
\hline Cantu [7] & $\begin{array}{l}\text { 1. No loss } \\
\text { of consciousness } \\
\text { OR } \\
\text { 2. Posttraumatic } \\
\text { amnesia, } \\
\text { signs/symptoms } \\
\text { last longer than } \\
30 \text { minutes }\end{array}$ & $\begin{array}{l}\text { 1. Loss of } \\
\text { consciousness lasts } \\
\text { less than } 1 \text { minute } \\
\text { OR } \\
\text { 2. Posttraumatic } \\
\text { amnesia lasts longer } \\
\text { than } 30 \text { minutes but } \\
\text { less than } 24 \text { hours }\end{array}$ & $\begin{array}{l}\text { 1. Loss of consciousness } \\
\text { lasts more than } \\
1 \text { minute } \\
\text { OR } \\
\text { 2. Posttraumatic amnesia } \\
\text { lasts longer than } \\
24 \text { hours } \\
\text { OR } \\
\text { 3. Postconcussion signs } \\
\text { or symptoms last } \\
\text { longer than } 7 \text { days }\end{array}$ \\
\hline
\end{tabular}

30 minutes. Same-day return to competition was allowed only if the athlete was completely asymptomatic following the injury.

Although the abovementioned management guidelines reached their zenith of popularity during the 1980 s and 1990 s, in the late 1990 s sports medicine practitioners and organizations began to question the scientific basis of these guidelines. This trend prompted the American Orthopaedic Society for Sports Medicine (AOSSM) to sponsor a workshop with the purpose of re-evaluating current guidelines and establishing practical alternatives [15]. Although the AOSSM guidelines did not differ substantially from prior guidelines, this workshop started a trend away from the use of a numeric grading systems for determination of return to play following concussion (eg, as developed by the Cantu, Colorado, and AAN guidelines). The AOSSM guidelines were also the 
first to stress more individualized management of injury, rather than applying general standards and protocols.

Yet another important development with regard to concussion management took place in 2002 under the auspices of the Federation Internationale de Football Association in conjunction with the International Olympic Committee and the International Ice Hockey Federation. The organizers of this meeting assembled a group of physicians, neuropsychologists, and sports administrators in Vienna, Austria, to continue to explore methods of reducing morbidity secondary to sports-related concussion. The deliberations that took place during this meeting lead to the publication of a document outlining recommendations for both the diagnosis and management of concussion in sports [16]. One of the most important conclusions of this meeting was that no previously published concussion management guidelines were adequate for assuring proper management of every concussion. Although a complete discussion of these recommendations are beyond the scope of this article, the group emphasized the implementation of postinjury neuropsychologic testing as the "cornerstone" of proper postinjury management and return to play decision making.

The recognition of neuropsychologic testing as a key element of the postconcussion evaluation process represented a particularly important development in the diagnosis and management of the concussed athlete. The use of baseline neuropsychologic testing was specifically recommended whenever possible. In addition, a graduated return to play protocol was emphasized. The specific recommendations of the concussion in sports (CIS) group are presented in Box 3. It

\section{Box 3. Vienna Concussion Conference: return to play recommendations}

Athletes should complete the following stepwise process prior to return to play following concussion:

\section{Removal from contest following any signs/symptoms} of concussion

2. No return to play in current game

3. Medical evaluation following injury

a. Rule out more serious intracranial pathology

b. Neuropsychologic testing (considered a cornerstone of proper postinjury assessment)

4. Stepwise return to play
a. No activity and rest until asymptomatic
b. Light aerobic exercise
c. Sport-specific training
d. Noncontact drills
e. Full-contact drills
f. Game play 
was specifically recommended that each step would, in most circumstances, be separated by 24 hours. Furthermore, any recurrence of concussive symptoms should lead to the athlete dropping back to the previous level. In other words, if an athlete is asymptomatic at rest and develops a headache following light aerobic exercise, the athlete should return to complete rest. The Vienna group further recognized that conventional structural neuroimaging studies (eg, CT, MRI, electroencephalography) are typically unremarkable following concussive injury and should be employed only when a structural lesion is suspected. The group further suggested that functional imaging techniques are in the early stages of development but may provide valuable information in the future (see later discussion).

\section{Individual factors determining return to play}

Once a concussed athlete has been removed from a game, the sports medicine physician is faced with the often challenging decision of when the athlete is safely able to return to play. Making return to play decisions, both on the field and during the postinjury management of injury (ie, days after injury) in athletes can be one of the most complex decisions facing the sports medicine physician and represents a dynamic process that involves the evaluation of factors such as the severity of the injury (as measured by duration loss of consciousness, amnesia, and confusion), the athlete's reported symptoms (eg, lingering headache, fatigue, photosensitivity) and performance on neurocognitive testing (see later discussion), and the athlete's prior concussion history. In addition to these variables that relate to clinical recovery, there are many other potential factors that may play a role in the decision-making process. Since the advent of neurocognitive testing, and more individualized management of injury, prospective data are emerging that shed light on individual factors that may play a role in the incidence, severity, and length of recovery regarding concussion.

Age

Current concussion guidelines assume identical return to play criteria for athletes regardless of age. Based on these guidelines, it has traditionally been assumed that the speed of recovery is the same at all age groups and athletic levels. Unfortunately, before 2003 there was no existing published study examining outcome from concussion in the high school athlete or younger populations. Recent research has begun to expose potential differential age-related responses to concussive injury.

Based on research done with more severe mild traumatic brain injury, several theories do exist that might explain age-related differences to recovery from concussion. One such theory is that children may undergo more prolonged and diffuse cerebral swelling after traumatic brain injury, which suggests that they may be at an increased risk for secondary injury [17]. Moreover, the immature brain may be up to 60 times more sensitive to glutamate [18], a neurotransmitter 
involved in the metabolic cascade following concussion. These factors may lead to a longer recovery period and could increase the likelihood of permanent or severe neurologic deficit should reinjury occur during the recovery period. Such a theory may help account for the finding that second impact syndrome has been found to occur only in adolescent athletes [8].

One published study [19] has directly examined recovery from concussion in college versus high school aged athletes. Specifically, baseline and postconcussion neurocognitive functioning was measured in a sample of 53 athletes. Even though the college sample had a greater prior incidence of concussion, high school athletes were found to take longer to recover during an in-study concussion. This study suggests more protracted recovery from concussion in high school athletes. Another recently published study [20] reveals the apparent heightened vulnerability to concussion in the high school athlete. Specifically, the issue of the "bell ringers" or very mild concussion was examined in high school athletes aged 13 to 17 years. This study revealed that high school athletes with less than 15 minutes of on-field symptoms required at least 7 days before full neurocognitive and symptom recovery. This study and a follow-up analysis [21] call into question the validity of the grading systems for management of mild concussion. These findings suggest that all high school athletes diagnosed with concussion during a contest should be removed from play.

Overall recommendations from the Vienna meeting also recommended that all concussed athletes be removed from a contest if concussion is diagnosed [16]. It should be stressed that no prospective data have examined the issue of grade 1 concussion in college- or professional-aged athletes. As noted above, there may be significant age-related differential response to concussive injury. Moreover, intangibles such as level of competition and overall risk/benefit analysis is also likely to be different at different levels (eg, professionals versus amateurs). Professional athletes may be willing to assume greater risk given obvious monetary and other considerations. Conversely, few parents would risk injury in a high school athlete who is unlikely to compete beyond high school.

\section{Concussion history}

In addition to age, the concussion history of an athlete may potentially be an important determinant for return to play. There is a growing body of evidence that suggests there may be cumulative detrimental effects of multiple concussions. This has typically been associated with the neuropsychologic impairment and neurologic abnormalities that have primarily been documented among boxers $[22,23]$. Lately, however, this topic has been of rising concern among other athletic populations. In a study of almost 400 college football players, Collins and colleagues [24] revealed long-term subtle neurocognitive deficits in players who suffered two or more concussions. Matser and colleagues [25] suggested that cumulative long-term consequences of repetitive blows to the head are being seen in professional soccer players. In addition, high school and collegiate athletes who suffer three or more concussions appear to be more vulnerable to subsequent 
injury than athletes who have no history of injury [26]. Even though data are emerging, there are simply no current reliable data available to determine how many concussions should preclude return to play or forced retirement from sport.

Yet another issue that is often evaluated in making return to play decisions is the amount of time between injuries. Although no current research has formally investigated this factor, most concussion management guidelines contain a provision for restriction of play following multiple concussions within a given season $[4,12,14,15]$. This recommendation appears to be based on the wellestablished clinical experience of many sports medicine physicians that recently concussed athletes are prone to reinjury if they suffer soon after an initial injury.

Given the complexity of multiple potential factors contributing to recovery and outcome following sports-related concussion, individualized evaluation is stressed for management of sports concussion. Over the past decade, neurocognitive testing has been repeatedly demonstrated as a sensitive, valid, and practical assessment tool for helping to make more quantified and data-driven decisions regarding postinjury concussion management.

\section{Neuropsychologic testing}

The development of neuropsychologic testing as a diagnostic tool in sports medicine is a relatively recent development that initially took place in the mid1980 s within the context of a large, multisite research project undertaken by Barth and colleagues [27] at the University of Virginia. This study demonstrated the potential use of neuropsychologic test procedures to document cognitive recovery within the first week following concussion. However, this study did not result in the widespread adoption of neuropsychologic testing in organized athletics at the clinical level. In the early 1990s, a series of events transpired that shifted the use of neuropsychologic testing in sports to the clinical arena. First, injuries to a number of high-profile professional athletes resulted in the implementation of baseline neuropsychologic testing by a number of National Football League (NFL) teams in the mid-1990s [28]. Similarly, following careerending injuries in the National Hockey League (NHL), the NHL mandated baseline neuropsychologic testing for all athletes [29]. In addition to the increased use of neuropsychologic testing in professional sports, several large-scale studies of collegiate athletes were undertaken. These studies [1,30-32] further verify that neuropsychologic testing yielded useful clinical information. Specifically, neuropsychologic testing allowed a baseline/postinjury analysis of the subtle aspects of cognitive function likely affected by concussive injury, thus providing objective data to make more informed decisions regarding return to play.

The use of traditional neuropsychologic testing (eg, paper and pencil testing) has resulted in a rapid expansion of our knowledge regarding concussions and has become a popular clinical tool within the professional ranks. However, the more widespread application of testing within the college ranks has been limited due to practical and economic constraints. Furthermore, neuropsychologic testing 
at the high school level has been extremely limited before the year 2000. This latter fact is disturbing given that the vast majority of at-risk athletes fall within the high school ranks and below. Traditional neuropsychologic testing has proven to be too time-consuming and costly for many amateur organizations, and the expansion of testing has also been limited by a shortage of trained neuropsychologists to oversee the administration and interpretation of the assessment process. As a result of these inherent limitations of traditional assessment and in parallel to the widespread proliferation of the microcomputer, several researchers have begun to develop computer-based neuropsychologic testing procedures.

Computer-based neuropsychologic testing procedures have a number of advantages and relatively few disadvantages when compared with more traditional neuropsychologic testing procedures. First, the use of computers allows the evaluation of large numbers of student athletes with minimal manpower. For example, through our program at the University of Pittsburgh, we routinely evaluate up to 20 athletes simultaneously within a high school or college computer laboratory. This promotes the assessment of an entire football team within a reasonable time period using minimal human resources. Second, data acquired through testing can be easily stored in a specific computer or computer network and can therefore be accessed at a later date (eg, following injury). Not only does this promote the efficient clinical evaluation of the athlete but also greatly expands the possibilities for research. Third, the use of the microcomputer promotes the more accurate measurement of cognitive processes such as reaction time and information processing speed. In fact, computerized assessment allows for the evaluation of response times that are accurate to the 1/100 of a second, while traditional testing allows for accuracy only to 1 to 2 seconds. This increased accuracy will no doubt increase the validity of test results in detecting subtle changes in neurocognitive processes. Fourth, the use of the computer allows for the randomization of test stimuli that should help to improve reliability across multiple administration periods, minimizing the "practice effects" that naturally occur with multiple exposures to the stimuli. These practice effects have clouded the interpretation of research studies and have also presented an obstacle for the clinician evaluating the true degree of neurocognitive deficit following injury. Limiting the influence of practice effects on testing allows a direct interpretation of postinjury data to the athlete's baseline to determine whether full cognitive recovery has occurred. Lastly, computer-based approaches allow for the rapid dissemination of clinical information into a coherent clinical report that can be easily interpreted by the sports medicine clinician. In summary, there are many benefits derived from a computerbased approach insofar as the technology has appropriate sensitivity, reliability, and validity to measuring the subtle aspects of concussive injury.

\section{Approaches to computer-based neuropsychologic testing}

Presently there are several computer-based management approaches under development to help provide the sports medicine clinician with neurocognitive 
data to better determine return to play and other management issues following concussive injury [33]. Specifically, four computer-based models have been detailed in the scientific literature. These include ImPACT (Immediate PostConcussion Assessment and Cognitive Testing) CogState, Headminders, and ANAM (Automated Neuropsychological Assessment Metric). Differences do exist between these test batteries and each is at different stages of validation. Clearly, issues such as sensitivity, reliability, and validity of the respective test batteries should be given careful scrutiny before implementation is adopted. In addition, issues regarding test selection, cognitive domains measured, details of the clinical report, and consultation options for each instrument should be given careful review before implementation. A detailed review of these critical issues for each computerized battery is beyond the scope of this article; however, readers are urged to adequately explore these germane areas before instituting this approach to injury management. Again, it is imperative that clinicians understand that the cognitive data derived from these instruments is not a panacea for concussion management. Rather, these tools provide one piece of clinical data for which to make complicated decisions regarding clinical management and eventual return to play options for the concussed athlete.

Computerized neuropsychologic testing is beginning to create heated interest within the sports medicine arena. The ability to collect objective, sensitive, and detailed neurobehavioral information pertaining to the athlete's postconcussive status is of obvious merit and transcends standard levels of care. For example, such an approach to injury is systematic and individually tailored, rather than applying general standards (eg, grading systems) to concussion management that lack empirical validation. To help familiarize the reader with a computerized approach to concussion management, we will review our concussion management program at the University of Pittsburgh Center for Sports Medicine.

\section{A clinical model for computerized neuropsychologic testing}

The University of Pittsburgh Medical Center (UPMC) Sports Medicine Concussion Program represents a specialized clinical and research program that uses neurocognitive testing to assist in general concussion management issues (eg, determining return to play, retirement decisions, and so forth). This program uses the ImPACT computer-based neuropsychologic testing program [10,20, 21,26]. This test battery was developed to address the inherent limitations of traditional neuropsychologic assessment and has allowed our program to provide consultative services to multiple colleges, high schools, and professional sports organizations. Although the development and construction of this computer program is detailed elsewhere [21,34], a brief review will be provided below. We should emphasize that other computer-based approaches to assessment are currently in use and our program represents only one such program.

The ImPACT neuropsychologic test battery is comprised of seven test modules that assess multiple neurocognitive abilities. In the interest of gathering as 
much neuropsychologic data within an approximate 20-minute administration time, several modules are designed to simultaneously evaluate multiple cognitive domains. To aid in the clinical interpretation of test performance, select module scores are combined to yield composite indices. These composite scores were constructed to better isolate test performance within the cognitive domains of reaction time, verbal memory, visual memory, processing speed, and impulse control.

As an adjunct to the neurocognitive scores provided by the clinical report, a symptom self-report inventory is also included within the ImPACT program. This 21-item scale (Table 2) [35] requires the athlete to subjectively rank symptoms such as headache, dizziness, photosensitivity, and so forth. Each symptom is rated on a scale of 0 (no complaint) to 6 (severe symptom). This allows for the direct comparison of postconcussion symptoms to preinjury symptom reporting and promotes a more comprehensive understanding of the recovery process for athletes, health care professionals, and other interested individuals (eg, parents and coaches). It is important to note that subjective symptoms and neurocognitive test results do not always correlate, and the evaluation of both aspects of recovery is essential.

Although the issue of recovery from concussive injury is complex, we recommend that an athlete be symptom-free and cognitively intact both at rest

Table 2

The postconcussion symptom scale

\begin{tabular}{|c|c|c|c|c|c|c|c|}
\hline \multirow{2}{*}{$\frac{\text { Symptom }}{\text { Headache }}$} & \multirow{2}{*}{$\begin{array}{l}\text { None } \\
0\end{array}$} & \multicolumn{2}{|c|}{ Minor } & \multicolumn{2}{|c|}{ Moderate } & \multicolumn{2}{|c|}{ Severe } \\
\hline & & 1 & 2 & 3 & 4 & 5 & 6 \\
\hline Nausea & 0 & 1 & 2 & 3 & 4 & 5 & 6 \\
\hline Vomiting & 0 & 1 & 2 & 3 & 4 & 5 & 6 \\
\hline Balance problems & 0 & 1 & 2 & 3 & 4 & 5 & 6 \\
\hline Dizziness & 0 & 1 & 2 & 3 & 4 & 5 & 6 \\
\hline Fatigue & 0 & 1 & 2 & 3 & 4 & 5 & 6 \\
\hline Trouble falling asleep & 0 & 1 & 2 & 3 & 4 & 5 & 6 \\
\hline Sleeping more than usual & 0 & 1 & 2 & 3 & 4 & 5 & 6 \\
\hline Sleeping less than usual & 0 & 1 & 2 & 3 & 4 & 5 & 6 \\
\hline Drowsiness & 0 & 1 & 2 & 3 & 4 & 5 & 6 \\
\hline Sensitivity to light & 0 & 1 & 2 & 3 & 4 & 5 & 6 \\
\hline Sensitivity to noise & 0 & 1 & 2 & 3 & 4 & 5 & 6 \\
\hline Irritability & 0 & 1 & 2 & 3 & 4 & 5 & 6 \\
\hline Sadness & 0 & 1 & 2 & 3 & 4 & 5 & 6 \\
\hline Nervousness & 0 & 1 & 2 & 3 & 4 & 5 & 6 \\
\hline Feeling more emotional & 0 & 1 & 2 & 3 & 4 & 5 & 6 \\
\hline Numbness or tingling & 0 & 1 & 2 & 3 & 4 & 5 & 6 \\
\hline Feeling slowed down & 0 & 1 & 2 & 3 & 4 & 5 & 6 \\
\hline Feeling mentally "foggy" & 0 & 1 & 2 & 3 & 4 & 5 & 6 \\
\hline Difficulty concentrating & 0 & 1 & 2 & 3 & 4 & 5 & 6 \\
\hline Difficulty remembering & 0 & 1 & 2 & 3 & 4 & 5 & 6 \\
\hline Visual problems & 0 & 1 & 2 & 3 & 4 & 5 & 6 \\
\hline
\end{tabular}

Adapted from Lovell MR, Collins MW. Neuropsychological assesstment of the college football player. J Head Trauma Rehab 1998;13:9-26; with permission. 
and following exertional activity before return to sport participation following concussive injury. Obviously, the optimal use of this approach is to compare an athlete to his or her own individual baseline. However, in the absence of baseline data this evaluative process is quite informative by comparing the athlete with extensive normative data that may serve as an adequate comparison to determine recovery.

\section{Other neurodiagnostic techniques}

Although neuropsychologic testing has increasingly been used as both as a research and clinical tool, the past several years have witnessed the emergence of an intense focus on the development of sophisticated brain imaging techniques. This interest grew out of new technologic advances in brain imaging in addition to frustration regarding the well-known lack of sensitivity of more traditional anatomic imaging techniques such as CT and MRI. Although useful in identifying brain injury in more severe cases, CT and MRI scans are not often helpful in identifying more subtle brain-related changes that are thought to occur on a metabolic rather than anatomic level [21].

For example, recent research has examined the potential use of functional magnetic resonance imaging (fMRI) as a viable tool for the assessment of neural processes following mild traumatic brain injury. The technology is based on the measurement of specific correlates of brain activation such as cerebral blood flow and oxygenation [36]. fMRI also promotes the study of brain activation (or lack thereof) through the "in scanner" implementation of specific neurocognitive tasks. The administration of specific neuropsychologic tests to nonconcussed individuals during the fMRI scanning process results in measurable and predictable changes in brain activity that can be contrasted with the performance of athletes following concussion. fMRI involves no exposure to radiation and can be safely used in children. Furthermore, repeat evaluations can also be undertaken with minimal risk. This promotes the assessment of changes in neural substrata that may occur with mild concussion and makes it possible to track the recovery process. Potentially, one of the most important uses of fMRI scanning is the ability to provide validity data regarding the sensitivity and specificity of neuropsychologic testing to detect subtle changes in brain function.

Although a promising tool, fMRI has yet to be widely implemented in clinical settings. This is no doubt a function of the sophisticated technology needed to acquire and analyze the images and the considerable expense of the procedure. Presently only a few laboratories are actively investigating the use of fMRI in sports-related head injury, although this is likely to change within the next few years. Notably, Johnston and colleagues [7] at McGill University have developed an fMRI protocol that allows the assessment of several components of working memory. In the United States, our laboratory at the University of Pittsburgh represents one of only a handful of research programs structured to collect both neuropsychologic and fMRI data in athletes. This multiyear prospective study 
relies on the prior baseline neuropsychologic testing (ImPACT) of a very large cohort (over 1500) of male and female high school and college athletes. In the event of an injury, the athletes will undergo repeat testing within 24 to 72 hours and will also undergo fMRI scanning. An additional fMRI scan as well as ImPACT testing "in scanner" and "out of scanner" will be completed as the patient recovers, allowing us to track the correlation between fMRI and neuropsychologic testing. Our initial pilot data have been highly encouraging and have established the resolution of fMRI abnormalities and deficits in memory, reaction time, and processing speed.

Fig. 1 provides a graphic depiction of the fMRI of a 14-year-old male athlete who suffered a concussion without loss of consciousness. Initial signs of injury did not include loss of consciousness or overt retrograde or anterograde amnesia. Rather, the patient exhibited a brief period of confusion (approximately $30 \mathrm{sec}-$ onds), with concomitant dizziness and mild photosensitivity. No emergent follow-up was conducted (eg, CT, MRI). The following morning, this individual complained of a right temporal headache, as well as mild fatigue and lassitude. He was referred to the UPMC Sports Concussion Program and was evaluated subsequently via both neuropsychological testing and an fMRI study. This image shows his initial fRMI data at 5 days after injury. His scan reveals an abnormal pattern of brain activation in brain areas during the NBACK working memory task. This is thought to reflect an increase in brain areas needed to complete the NBACK task following concussive injury [36]. The patient was also evaluated 28 days postinjury. The subject's fMRI scan at this time had normalized as

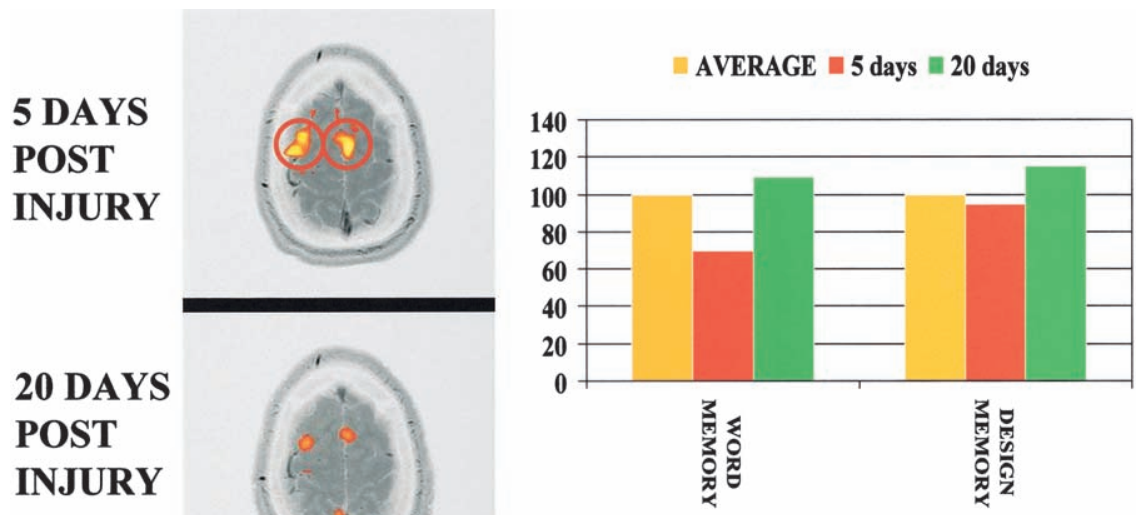

Average score is $100(\mathrm{SD}=15)$

Fig. 1. fMRI and ImPACT case study data. fMRI and ImPACT data are presented for a 17-year-old motocross rider without loss of consciousness. At left, increased fMRI activation is shown at 48 hours post-injury which resolves in a follow-up evaluation at 20 days post-trauma. At right, ImPACT deficits (relative to baseline) are noted at 5 days post-concussion in terms of memory. The patient also reports an increase in symptoms. Resolution of ImPACT deficits are also evidenced at 20-days post-concussion. 
evidenced by activation of expected brain areas in the frontal and temporal lobes. His neuropsychologic test results (ImPACT) were congruent with this resolution of abnormal patterns visible through fMRI, demonstrating initial decline at 5 days after injury and a return to prebaseline levels at 20 days postinjury.

\section{University of Pittsburgh Medical Center Sports Concussion Program: protocol for return to play}

Based on recent research regarding recovery from concussion and the Vienna international consensus statement [16], the UPMC return to play protocol involves the graduated return to play of the athlete to competition based on his or her progression through several steps in the recovery process. First and foremost, we feel strongly that athletes who have either abnormal neuropsychologic test results or are symptomatic should not be returned to play following injury until they are asymptomatic and any cognitive difficulties have resolved.

As mentioned previously, we also strongly suggest that younger athletes (eg, high school-age and younger) should not be returned to play during the game in which they were injured. This allows for closer evaluation of evolving signs and symptoms and will help to prevent more severe injury. We recommend formal neuropsychologic testing (eg, ImPACT) the day after injury to assess initial neurocognitive status. It is also important to re-evaluate the athlete regularly with regard to reported at-rest symptoms.

Once the athlete is symptom-free and at rest, we suggest graduated aerobic exertional testing to check for the return of symptoms such as headache, dizziness, nausea, or fogginess. When the athlete becomes asymptomatic following exertion, neuropsychologic testing should also be completed and the athlete's test results should be compared with their baseline. If preinjury baseline neuropsychologic testing has not been previously completed, the athlete's test performance should be compared with normative standards for their age and gender. Finally, it should be emphasized that this protocol is based on research with younger, primarily high school-aged subjects and the management of the older athlete (eg, college-aged or professional) may vary. Hopefully, recent large-scale research projects conducted by the NFL and NHL will shed additional light on the management of concussion in professional athletes.

\section{Conclusions and future directions}

This article has focused on new developments in the management of sportsrelated concussion. Specifically, we have emphasized that the clinical management of concussion is evolving rapidly, and we still have much to learn about both the short- and long-term consequences of injury. As increasingly more research studies are designed to investigate the biomechanics, pathophysiology, and clinical course of sports-related concussion, our management strategies will 
continue to evolve over the next 5 to 10 years. Although the future of concussion management remains somewhat uncertain, one trend has become particularly clear over the past 2 years: concussion management has become increasingly individualized. This trend is likely to continue. The 1980s and 1990s were characterized by the publication of multiple concussion guidelines that made specific return to play recommendations based on duration of concussion markers such as loss of consciousness, amnesia, and so forth. However, there has been recent acknowledgment by leaders in the field that guidelines may have limited value, and the focus has shifted to a more individualized approach based on the establishment of an absence of any clinical symptomatology and establishment of normal brain function before return to play. The diagnostic techniques outlined in this article will likely play an important role in the clinical management of concussion.

\section{References}

[1] Collins MW, Lovell MR, McKeag DB. Current issues in managing sports concussion. JAMA 1999;282:2283-5.

[2] Grindel SH, Lovell MR, Collins MW. The assessment of sports-related concusions: the evidence behind neuropsychological testing and management. Clin J Sport Med 2001;11:134-43.

[3] Congress of Neurological Surgeons. Committee on head injury nomenclature: glossary of head injury. Clin Neurosurg 1966;12:386-94.

[4] American Academy of Neurology. Practice parameter: the management of concussion in sports (summary statement). Report of the Quality Standards Subcommittee. Neurology 1997;48:581-5.

[5] Hovda DA, Prins M, Becker DP. Neurobiology of concussion. In: Bailes JE, Lovell MR, Maroon JC, editors. Sports-related concussion. St. Louis (MO): Quality Medical Publishing; 1999. p. $12-51$.

[6] Bergschneider M, Hovda DA, Shalmon E. Cerebral hyperglycolsis following severe human traumatic brain injury: a positron emission tomography study. J Neurosurg 2003;86:241-51.

[7] Johnston KM, Pitto A, Chankowsky I, Chen JK. New frontiers in diagnostic imaging in concussive head injuries. Clin J Sport Med 2001;11:166-75.

[8] Cantu R, Voy R. Second impact syndrome: a risk in any sport. Phys Sportsmed 1995;23:27-36.

[9] Collins MW, Field M, Lovell MR, Iverson GL, Johnston KM, Maroon J, et al. Relationship between post-concussion headache and neuropsychological test performance in high school athletes. Am J Sports Med 2003;31:168-73.

[10] Collins MW, Iverson GL, Lovell MR, McKeag DB, Norwig J, Maroon JC. On-field predictors of neuropsychological and symptom deficit following sports-related concussion. Clin J Sport Med 2003;13:222-9.

[11] Erlanger D, Kausik T, Cantu R, Barth JT, Broshek DK, Freeman JR, et al. Symptom-based assessment of the severity of concussion. J Neurosurg 2003;98:34-9.

[12] Cantu RC. Cerebral concussion in sport: management and prevention. Phys Sportsmed 1992; $14: 64-74$.

[13] Kelly JP, Nichols JS, Filley CM. Concussion in sports: guidelines for the prevention of catastrophic outcome. JAMA 1991;266:2867-9.

[14] Cantu RC. Posttraumatic retrograde and anterograde amnesia: pathophysiology and implications in grading and safe return to play. J Athletic Training 2001;36:244-8.

[15] Wojyts ED, Hovda D, Landry G, Boland A, Lovell MR, McCrea M, et al. Concussion in sports. Am J Sports Med 1999;27:676-86. 
[16] Aubry M, Cantu R, Dvorak J, Graf-Bauman T, Johnston KM, Kelly J, et al. Summary of the first international conference on concussion in sport. Clin J Sports Med 2002;12:6-11.

[17] Pickles W. Acute general edema of the brain in children with head injuries. N Engl J Med 1950; 242:607-11.

[18] McDonald JW, Johnston MV. Physiological and pathophysiological roles of excitatory amino acids during central nervous system development. Brain Res Brain Res Rev 1990;15:41-70.

[19] Field M, Collins MW, Lovell MR, Maroon JC. Does age play a role in recovery from sportsrelated concussion? A comparison of high school and collegiate athletes. J Pediatr 2003;142: 546-53.

[20] Lovell MR, Collins MW, Iverson GL, Field M, Maroon JC, Cantu R, et al. Recovery from mild concussion in high school athletes. J Neurosurg 2003;98:296-301.

[21] Lovell MR, Collins MW, Iverson GL, Johnston KL, Bradley J. Grade 1 or “ding” concussions in high school athletes. Am J Sports Med 2004;32(1):47-54.

[22] Jordan BD, Relkin NR, Ravdin LD. Apolipoprotein E e4 associated with chronic traumatic brain injury in boxing. JAMA 1997;278:136-40.

[23] Roberts GW, Allsop B, Bruton C. The occult aftermatch of boxing. J Neurol Neurosurg Psychiatry $1990 ; 53: 373-8$.

[24] Collins MW, Grindel SH, Lovell MR, Dede DE, Moser DJ, Phalin BR, et al. Relationship between concussion and neuropsychological performance in college football players. JAMA 1999;282:964-70.

[25] Matser E, Kessels A, Lezak M. Neuropsychological impairment in amateur soccer players. JAMA 1999;282:971-4.

[26] Collins MW, Lovell MR, Iverson GL, Cantu RC, Maroon JC, Field M. Cumulative effects of sports concussion in high school athletes. Neurosurg 2002;51:1175-81.

[27] Barth JT, Alves WM, Ryan TV, Macciocchi SN, Rimel RW, Jane JA. Mild head injury in sports. In: Levin HS, Eisenberg H, Benton A, editors. Mild head injury. Oxford (UK): Oxford University Press; 1989. p. 257-75.

[28] Lovell MR. Evaluation of the professional athlete. In: Bailes JE, Lovell MR, Maroon JC, editors. Sports-related concussion. St. Louis (MO): Quality Medical Publishing; 1999. p. 200-14.

[29] Lovell MR, Burke CJ. Concussion management in professional hockey. In: Cantu RE, editor. Neurologic athletic head and spine injury. Philadelphia: WB Saunders; 2000. p. 109-16.

[30] Echemendia RJ, Putukian M, Macklin RS. Neuropsychological test performance prior to and following sports-related mild traumatic brain injury. Clin J Sport Med 2001;11:23-31.

[31] Hinton-Bayre AD, Geffen GM, Geffen LB. Concussion in contact sports: reliable change indices of impairment and recovery. J Clin Exp Neuropsychol 1999;21:70-86.

[32] Macciocchi SN, Barth JT, Alves W. Neuropsychological functioning and recovery after mild head injury in collegiate athletes. Neurosurg 1996;39:510-4.

[33] Schnirring L. How effective is computerized concussion management? Phy Sportmed 2001; 29:11-6.

[34] Maroon JC, Lovell MR, Norwig J. Cerebral concussion in athletes:evaluation and neuropsychological testing. Neurosurg 2000;47:659-72.

[35] Lovell MR, Collins MW. Neuropsychological assessment of the college football player. J Head Trauma Rehab 1998;13:9-26.

[36] McAllister TW, Sparling MB, Flashman LA. Differential working memory load effects after mild head injury. Neuroimage 2001;14:1004-12. 